Sharif University of Technology
Scientia Iranica
SCIENTIA
I RAN ICA
http://scientiairanica.sharif.edu

\title{
Effects of the first reinforcement depth on different types of geosynthetics
}

\author{
E. Cicek ${ }^{a, *}$, E. Guler ${ }^{b}$, and T. Yetimoglu \\ a. Department of Civil Engineering, Hacettepe University, Ankara, Turkey. \\ b. Department of Civil Engineering. Bogazici University, Istanbul, Turkey. \\ c. Department of Civil Engineering, Ataturk University, Erzurum, Turkey.
}

Received 3 December 2016; received in revised form 25 April 2017; accepted 15 July 2017

\section{KEYWORDS}

First reinforcement depth;

Settlement;

Reinforcement;

Sand soil;

Geosynthetic;

Plate load test.

\begin{abstract}
This study investigates the performance of the first reinforcement layer depth for sand subbase of a road or construction by plate load laboratory tests. Unreinforced and reinforced experiments on different reinforcement types were made by changing depth ratio of the first reinforcement layer. One type of geotextile and two different geogrid specimens were used in the research. 'Load-settlement curves' and 'Bearing Ratios' were studied by measuring the results of different settlement ratios. Finally, laboratory measurements of unreinforced and reinforced soils using geotextile reinforcement were compared with FiniteElement Model (FEM) analyses modeled in similar conditions. The results demonstrated the effects of different types of reinforcements for different first reinforcement layer locations. The number of reinforcement layers was another parameter that affected the bearing ratio along with the first reinforcement layer depths. It was also observed that the bearing ratio (BR) and load-settlement behavior changed significantly with the first reinforcement depth and settlements. Effects on failure modes for unreinforced and reinforced sand soils were compared for each test.

(C) 2019 Sharif University of Technology. All rights reserved.
\end{abstract}

\section{Introduction}

Subbase soil is a significant element of the design of any structure, and improvement of bearing capacity and reduction of settlement can be achieved by making use of materials such as geosynthetic reinforcement. Geosynthetic reinforcement is used successfully in stabilization of soils, leading to an increase in bearing properties and a decrease in the settlement of footings, railway and highway subgrades, embankments, etc. As is known from the literature, reinforcements can extend the

*. Corresponding author. Tel.: +90 3122977333 E-mail addresses: elif.cicek@hacettepe.edu.tr, elifflower25@gmail.com (E. Cicek), eguler@boun.edu.tr (E. Guler),yetimt@atauni.edu.tr(T. Yetimoglu).

doi: $10.24200 /$ sci. 2017.4231 service span for pavements [1-6]. Additionally, in recent years, many researchers [7-13] have investigated the behavior of reinforced subgrades. It is well known that the performance of the reinforced systems is enhanced mainly because of the reinforcement properties such as position [14,15], strength [16], and mesh size $[17,18]$ of the reinforcement. The location of reinforcement material within the base layer is very critical for its effect [19]. However, when a detailed literature survey is conducted, it can be seen that there is no unique value for the optimum first reinforcement depth. To illustrate this fact, the following literature citations are helpful which are presented in summary as follows. Al-Qadi et al. [20] reported that, for a thin dense layer, placing geogrid material on it can have better improvement and that the reinforcement should be located at the upper one-third part of the base for a thicker layer. Nevertheless, Hass et al. [21] also found 
that effects did not last long when a single layer of reinforcement was at the midpoint or higher within the base layer for a thick base course over very soft, flexible subgrades. In order to clarify this issue, in this study, different first reinforcement depth values were chosen to investigate.

It is a known fact that the geosynthetic properties play a major role in the behavior. However, in most studies, one geosynthetic type was used. There is only limited information in the literature on the effect of different reinforcement types. Guido et al. [22] and Chen [23] investigated the reinforcement types, and concluded that geogrids showed superior performance compared to geotextiles. Mandal and Manjunath [24] investigated the bearing capacity behavior of Geogrid and bamboo stick reinforcements for strip plates on dense sand. A great increase in bearing capacity occurred when the reinforcement materials were placed within the distance of $0.5 \mathrm{~B}$. The geogrid materials had greater effect than bamboo materials did. Dash et al. [25] studied the measurements taken from laboratory experiments for strip plates on reinforced sand. Tensile strength of the reinforcement was not an important argument to compute the effect of the reinforcement. Aperture size and orientation of the ribs for geogrid material were significant contributions for the load-carrying mechanism. Alamshahi and Hataf [26] conducted experiments and numerical studies to determine the bearing capacity of a strip plate on reinforced slopes. The effects of the geogrid reinforcements and their locations were investigated. However, all studies show a lack of different reinforcement types' behavior on optimum first reinforcement depth and different settlement ratios. For this reason, in this study, different geosynthetic types with different mesh sizes were used to determine the load-settlement behavior of reinforced sand soil.

Most of the tests reported in the literature have been conducted on dense sand; generally, small settlement ratios were observed before failure. However, it is known that the bearing failure mode also depends on the density of the soil. However, in the case of looseto-medium dense sand, larger settlements are observed and, during this process, the soil gradually gets denser, too. As a consequence, the behavior observed for different settlement ratios differs. Accordingly, there is a need to analyze the results of different settlement ratios for different types of reinforcement. Therefore, one of the aims of this paper is to determine the behavior of medium dense soil reinforced with different types of geosynthetics. Based on reports derived from the respective literature, the depth of the first reinforcement is a critical parameter that affects the behavior of reinforced soil foundations. To determine the effective reinforced zone depth, the first reinforcement depth is a very significant factor due to the improving behavior.
However, there is no one value to determine the effective behavior, and different studies show different optimum depth ratios. Therefore, this study devotes special emphasis to understanding how the depth of the first reinforcement layer affects the behavior. As is clear from the literature, the most effective depth ratios were selected to define the optimum value. Tests were conducted with a geotextile and two different geogrids. It is very common that multiple layers of reinforcement are used in practice. Therefore, the effect of the first layer of reinforcement was investigated for multiple reinforcement layers. Because the behavior changes with increasing settlement values as explained above, results were analyzed for different settlements. The experimental results were also compared using finiteelement model.

\section{Methodology}

\subsection{Material properties}

One type of sand was used in the experiments, and the specific gravity of the dry soil was 2.65. The coefficient of uniformity, the coefficient of curvature, and the effective particle size were $2.5,1$, and $0.22 \mathrm{~mm}$, respectively. The maximum dry unit of weight was $16.5 \mathrm{kN} / \mathrm{m}^{3}$, and minimum dry unit weight was $13.9 \mathrm{kN} / \mathrm{m}^{3}$. The unit weight and relative density of the sand were kept constant. Their values were $15 \mathrm{kN} / \mathrm{m}^{3}$ and $46 \%$. Subbase soil was layered with 20 or $25 \mathrm{~mm}$ into the test tank. Laboratory tests, such as standard triaxial compression tests, were made to determine the soil behaviors, and friction angle was found as 38 degree. The sand used in model tests can be classified as 'poorly graded' for Unified Soil Classification System and A-1-b for American Association of State Highway and Transportation Official's classification system. In this study, three different geosynthetic materials were employed, one of which is a woven geotextile and two different types of geogrids. The characteristics of each reinforcement material are shown in Table 1.

\subsection{Test set up}

The boundary conditions of the test tank were selected, such that it would not affect the tests. The properties of the test models were selected based on literature researches. Additionally, some finite-element analyses were conducted prior to the laboratory tests to find the true boundary conditions of the models. Test model is shown in Figure 1. A steel tank $(100 \mathrm{~cm} \times 50$ $\mathrm{cm} \times 100 \mathrm{~cm})$ was considered. Undesirable movements of the box were restrained by steel I profiles. The tests were conducted in plane strain conditions. To investigate the effects of the improved subbase on roads or foundation soil, loads were applied to a strip steel plate with the width of $10 \mathrm{~cm}$ ( $B=$ footing width) and thickness of $25 \mathrm{~mm}$. Loading was applied to 
Table 1. Properties of geosynthetic reinforcements.

\begin{tabular}{cccc}
\hline \multirow{2}{*}{ Property } & \multicolumn{3}{c}{ Type of geosynthetic material } \\
\cline { 2 - 4 } & Geotextile & Geogrid 1 & Geogrid 2 \\
\hline Material property & Polypropilen & Polyester & Polyester \\
Type & Woven & - & - \\
Ultimate tensile strength $(\mathrm{kN} / \mathrm{m})$ & 60 & 35 & 55 \\
Failure strain $(\%)$ & 16 & 8.5 & 12 \\
Mass per unit area $\left(\mathrm{g} / \mathrm{m}^{2}\right)$ & 310 & 220 & 300 \\
Aperture size $(\mathrm{mm})$ & - & $20 \times 20$ & $40 \times 40$ \\
\hline
\end{tabular}

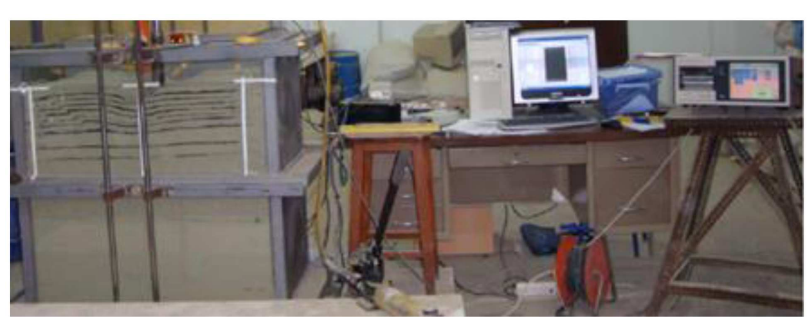

Figure 1. Test set-up.

settlement of plate equal to about $100 \%$ of $B$. The settlements of the soil were measured using four laser sensors displacement gauges. Two gauges were used and placed on the centre of each length. Additionally, in order to control the settlement results, four laser sensors were used and placed on four corners. Then, the results of the laser sensors and the gauges were the same. The loading values were measured by a load cell.

The geometrical parameters, describing the location of the reinforcement, are as follows: depth of the first reinforcement layer ' $u$ ', the vertical spacing of reinforcements ' $h$ ', the total number of reinforcement layers ' $N$ ', and the width of the geosynthetic reinforcement ' $L$ '. Several researchers have investigated the effect of the lengths of reinforcement [27-29]. They concluded that the optimum reinforcement length is $L=3 B$, because greater lengths are not efficient. As a result, the reinforcement length in this study was kept constant at $L=3 \mathrm{~B}$. A similar process was followed to determine the range of vertical spacing of reinforcement layers $(h)$ that gives the maximum bearing capacity, according to the results of a literature survey [30]. Therefore, the vertical spacing of reinforcement layers for multi-layered reinforced soil tests was taken as $h=0.4 B$ in all tests [10]. For this study, the number of reinforcement layers was chosen as follows: $N=0,1,2$, and 3 . To consider the effect of the first reinforcement depth $(u)$, the values of ' $u$ ' were chosen which are obtained from literature studies indicating optimum values, which are $u=0.175 B, 0.35 B, 0.55 B$, and $0.75 B$. The variables chosen for the tests are shown in Table 2 .

Initially, unreinforced models were made, and
Table 2. Variables chosen for the laboratory model tests of reinforced foundation soils.

\begin{tabular}{ccccc}
\hline $\boldsymbol{N}$ & $\boldsymbol{h} / \boldsymbol{B} \boldsymbol{L} / \boldsymbol{B}$ & $\boldsymbol{u} / \boldsymbol{B}$ & $\begin{array}{c}\text { Reinforcement } \\
\text { type }\end{array}$ \\
\hline 0 & - & - & - & Unreinforced \\
1 & - & 3 & $0.175,0.35,0.55$ & Geotextile reinforced \\
2 & 0.4 & 3 & $0.175,0.35,0.55,0.75$ & Geotextile reinforced \\
3 & 0.4 & 3 & $0.35,0.55,0.75$ & Geotextile reinforced \\
3 & 0.4 & 3 & $0.35,0.55,0.75$ & Geogrid 1 reinforced \\
2 & 0.4 & 3 & $0.35,0.55$ & Geogrid 2 reinforced \\
3 & 0.4 & 3 & $0.35,0.55,0.75$ & Geogrid 2 reinforced \\
\hline
\end{tabular}

then reinforced soil model tests were conducted. To compare the differences reported from the tests, loadsettlement curves and Bearing Ratio $(B R)$ values were used $(B R=q / q u$, where $q u$ is the contact pressure on plate in the unreinforced condition at a settlement ' $s$ ' and $q$ is the pressure for reinforced soil at the same settlement value ' $s$ ').

\subsection{Finite-element model properties}

Finite-element analyses were conducted to compare the laboratory test results and determine the reason for different behaviors under different reinforced conditions. The plane strain conditions were used and laboratory test conditions were simulated. To reduce the boundary effects, a detailed survey was made before the laboratory tests, and some numerical analyses were carried out to determine the model of the study. Therefore, a negligible level was chosen, and the width of the test tank was determined to be 10 times bigger, according to loading plate width $(B)$. In order to conduct a highly sensitive computation, the mesh size was further refined in the near vicinity of the plate and 15-node triangular elements were used. Five-node beam elements were used to model the plate, whose properties include $E I$ (flexural rigidity) $=260 \mathrm{kNm}^{2}$ $/ \mathrm{m}$ and $E A$ (axial stiffness) $=5 \times 106 \mathrm{kN} / \mathrm{m}$. The vertical boundaries were chosen to have only horizontal fixity and the lower boundary to have both horizontal and vertical fixities. The system was symmetric with 
Table 3. FEM soil properties.

\begin{tabular}{lccc}
\hline \multicolumn{1}{c}{ Parameter } & Sign & Unit & Value \\
\hline Unit weight & $\gamma$ & $\mathrm{kN} / \mathrm{m}^{2}$ & 15 \\
Elasticity modulus & $\mathrm{E}_{u r}$ & 25000 & $\mathrm{kN} / \mathrm{m}^{2}$ \\
Poisson ratio & $\nu$ & - & 0.25 \\
Cohesion & $c$ & $\mathrm{kN} / \mathrm{m}^{2}$ & 1 \\
Friction angle & $\varphi$ & Degree & 38 \\
Dilatancy angle & $\psi$ & Degree & 8 \\
& & & \\
$\begin{array}{l}\text { Reference stress } \\
\text { for stiffnesses }\end{array}$ & $P^{\text {ref }}$ & $\mathrm{kN} / \mathrm{m}^{2}$ & 100 \\
& & & \\
$\begin{array}{l}\text { Power for stress-level } \\
\text { dependency of stiffness }\end{array}$ & & & \\
\hline
\end{tabular}

the center of plate; therefore, only half of the system was modelled, as shown in literature studies [31]. The subbase soil was modelled by using hardening soil model, and the soil properties are shown in Table 3.

The cohesion was taken as $c=1 \mathrm{kN} / \mathrm{m}^{2}$ due to the plaxis finite-element program recommendation for sand soils. Dilatancy angle was obtained from $\varphi-30$ as recommended by the Plaxis manual. The elasticity module is secant elasticity modulus for hardening soil model. However, for reinforcement, only geotextile was modeled since the apertures of a geogrid were not possible to represent correctly by a $2 \mathrm{D}$ analysis, because only interface as a friction rate between soil and reinforcement material and elastic rigidity ( $E A)$ can be determined for reinforcement materials in the program. Elastic rigidity $(E A)$ was used to determine the geosynthetic mechanical behaviour. $E$ is the material tension stiffness, and ' $A$ ' represents the crosssectional areas for each reinforcement. Axial stiffness is similar to that in the literature studies. In this study, $E A=550 \mathrm{kN} / \mathrm{m}$ is taken for reinforcement, and interfaces between soil and geotextiles were considered as 0.7. This aforementioned data were selected through laboratory tests and adopted from manufacturing firms of the geotextiles.

\section{Results}

\subsection{Test results}

The load-settlement curve of the unreinforced soil is given in Figure 2. As can be seen in the figure, the failure surfaces started to develop in the soil when the load value reached $q u=61 \mathrm{kPa}$ and the settlement ratio $(s / B)$ was approximately $10-15 \%$. After failure, the curve becomes steeper. Hence, it can be interpreted that the soil behaves in an elastic manner before failure, and the plastic deformations prevail after the yield point. The soil is medium dense, and the measured

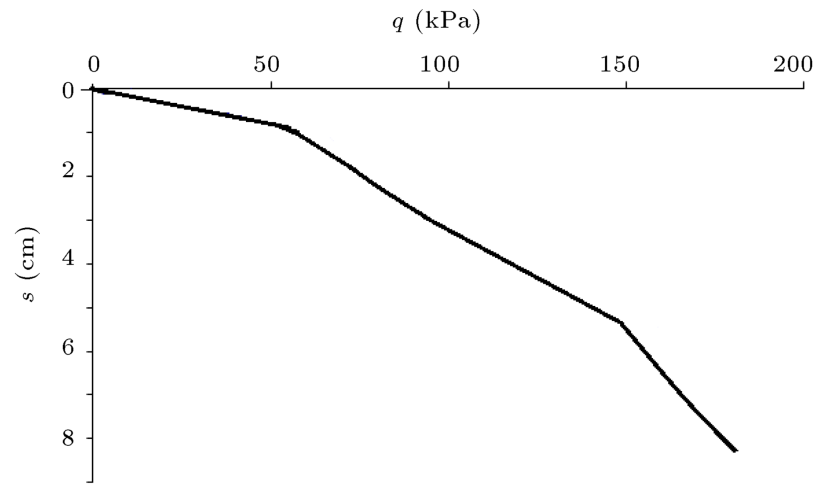

Figure 2. Load-settlement behavior of unreinforced sand.

deformations are the results of not only the elastic, but also plastic behaviors. The tests were repeated and similar results were obtained. The number of reinforcement layers was chosen as follows: $N=1,2$, and 3 for geotextile reinforced models. The first reinforcement depth was chosen as follows: $u=0.175$, $0.35,0.55$, and 0.75 based on literature studies. The load $(q)$-settlement $(s)$ results of measured smaller and larger settlement ratios are given in Figure 3. For a single reinforcement layer, the load-settlement curves indicate a failure point (Figure 3(a)). However, as the reinforcement layers add $(N \geq 2)$, a clear failure point can no longer be observed even for large settlement values (Figure 3(b) and (c)). As the first reinforcement depth ratios increase, the bearing ratio values of the reinforced soils become greater. For multi-layered soil at small settlement ratios, the bearing ratio increases up to the first reinforcement depth of $u / B=0.75$. At greater settlement ratios (approximately $s / B>0.2$ ), suddenly, the load-settlement behavior of multi-layered soils changes. The load value of the plate suddenly decreases; however, after this decrease, the plate starts to take up loads again. Generally, the maximum bearing ratio is observed for the first reinforcement depth of $u / B=0.55$. Small and big settlement ratios for one and three reinforcement layers show different behaviors. As the settlement ratio increases, $B R$ values also increase. This behavior is interpreted as the reinforcement taking more load. Additionally, as the settlements increase, the bearing ratio increases consequently.

The same tests were repeated with geogrid reinforcements to illustrate the difference. Two types of geogrids were used. In this series of the experiments, the first reinforcement depth was taken as $u / B=0.35$, 0.55 , and 0.75 . The value of $u / B=0.175$ was not used in this series, because, at such a shallow depth, the failing soil pushes the reinforcement beyond the loading plate to the surface. Additionally, the desired anchorage cannot be achieved due to the model scale, which is not to be expected in real structures. Furthermore, there is a sudden change and decrease in 


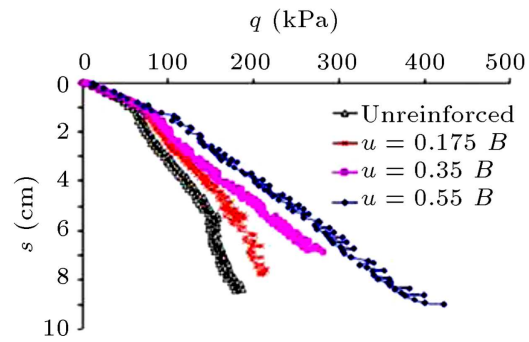

(a)
$q(\mathrm{kPa})$

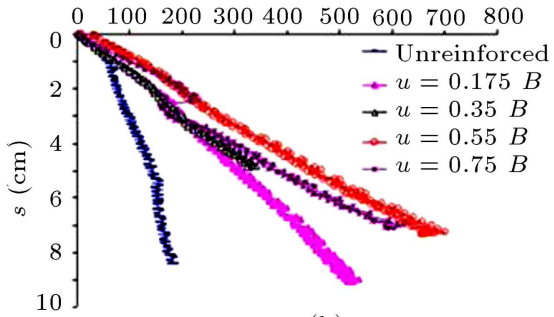

(b)

$q(\mathrm{kPa})$

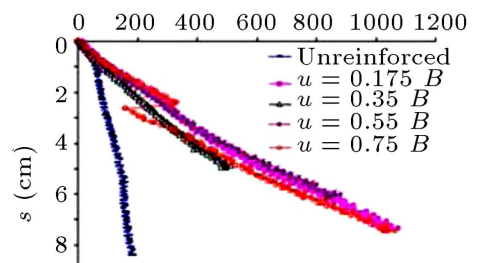

(c)

Figure 3. Load $(q)$-settlement (s) behavior of strip plate on geotextile reinforced sand for different first reinforcement depths: (a) $N=1$, (b) $N=2$, and (c) $N=3$.

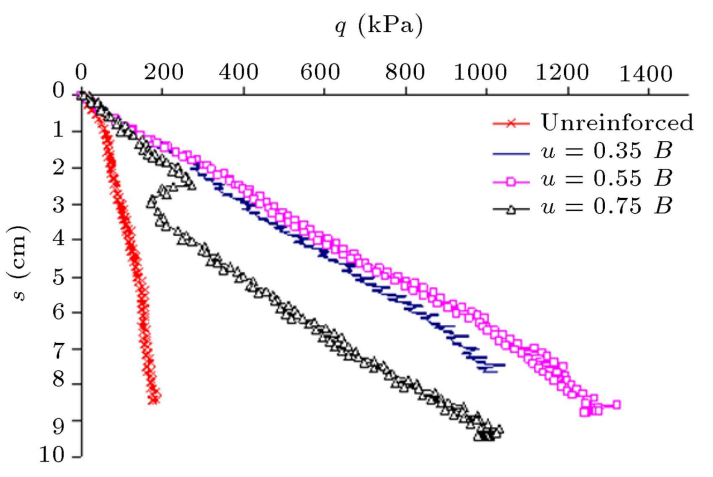

Figure 4. Load-settlement behavior of the first reinforcement depths for Geogrid 1 reinforced soil.

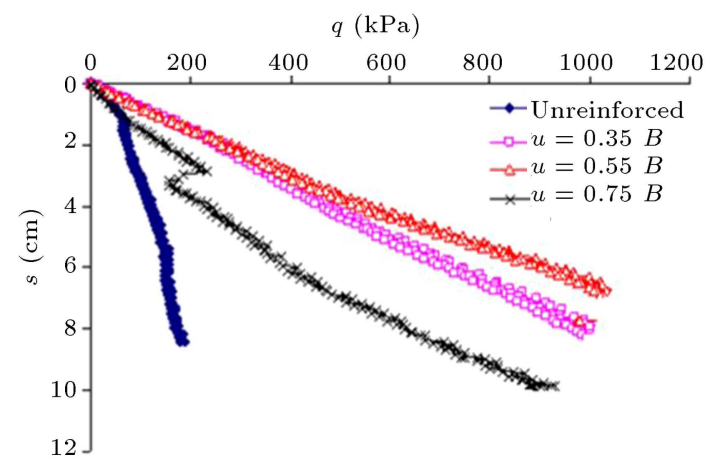

Figure 5. Load-settlement behavior of the first reinforcement depths for Geogrid 2 reinforced soil.

the load-carrying capacity in the experiments, where the first reinforcement depth is greater than 0.75 . Hence, the larger reinforcement depths were not used. Figures 4 and 5 show the load $(q)$-settlement $(s)$ results at different settlement ratios for soils reinforced with geogrids. It can be seen from Figures 4 and 5 that the maximum $B R$ values were generally measured for $u / B=0.55$ for both geogrid types. However, the first reinforcement depths give approximately the same $B R$ values at small settlement ratios for $u / B=0.35$ and 0.55 . As the settlement ratios increase, bearing ratio values also increase. The load decreases suddenly as in the geotextile reinforced model. The load-settlement curves of $u / B=0.35$ and 0.55 show a linear increase in $B R$ values, as the settlement ratio increases.

To determine the effect of different reinforcement types with various first reinforcement depths, the bearing ratio values were compared. In Figure 6 , the results of $N=3$ are given at different settlement ratios. The different reinforcement types showed different behaviors, as expected. For both geogrid reinforcements, similar behavior was observed. However, the behavior was different from that obtained for geotextile reinforcement. Generally, geogrid 1 reinforcement increases the bearing ratio values more than the other reinforcement types do, at $s / B \geq 0.2$. However, the geotextile reinforced model has bigger $B R$ values than others do, at $u / B=0.75$ and $s / B \geq 0.1$. Additionally, the $B R-u / B$ curve of the geotextile reinforced system behaves differently from the geogrids. Its curve has similar behavior at greater settlement ratios $(s / B \geq$ $0.3)$. Generally, the first reinforcement depth ratio, which is $u / B=0.55$, has greater bearing ratio values than the others do.

Most studies for $u / B=1$ in the literature reported that reinforcement could not increase the bearing capacity. Hence, $u / B=1$ was not used in this study. As can be seen in Figure 7, considering the load-settlement behaviors of reinforced medium dense sand for $u / B=0.75$, different geosynthetic types show a similar effect at the same settlement ratio. The load 


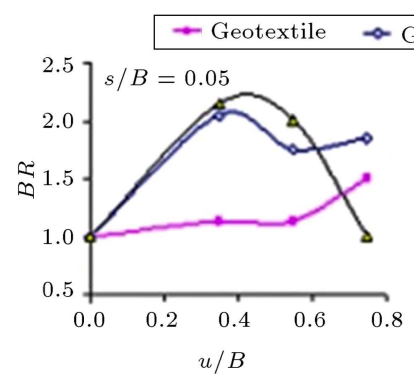

(a)

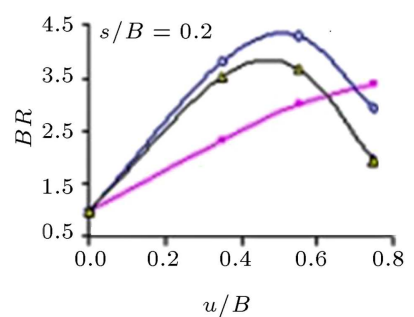

(c)

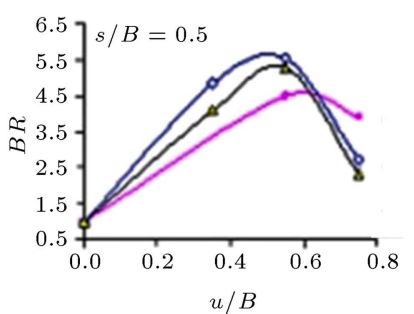

(e)

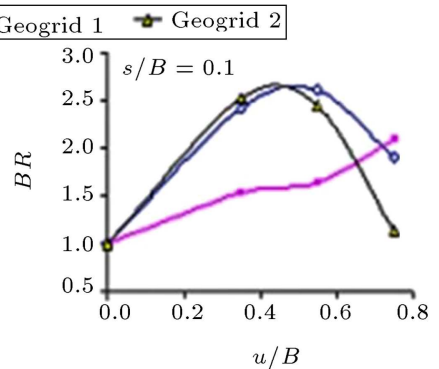

(b)

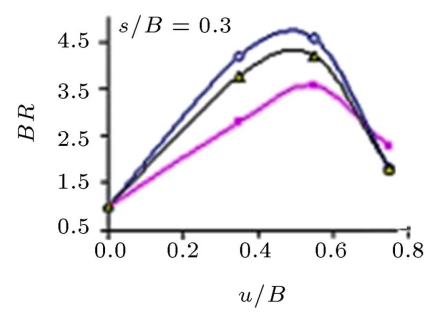

(d)

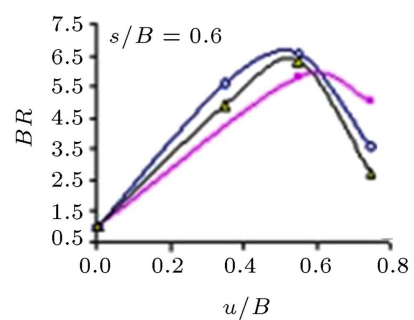

(f)
Figure 6. Comparison of different reinforcement types to investigate behavior of $B R-u / B$ : (a) $s / B=0.05$, (b) $s / B=0.1$, (c) $s / B=0.2$, (d) $s / B=0.3$, (e) $s / B=0.5$, and (f) $s / B=0.6$.

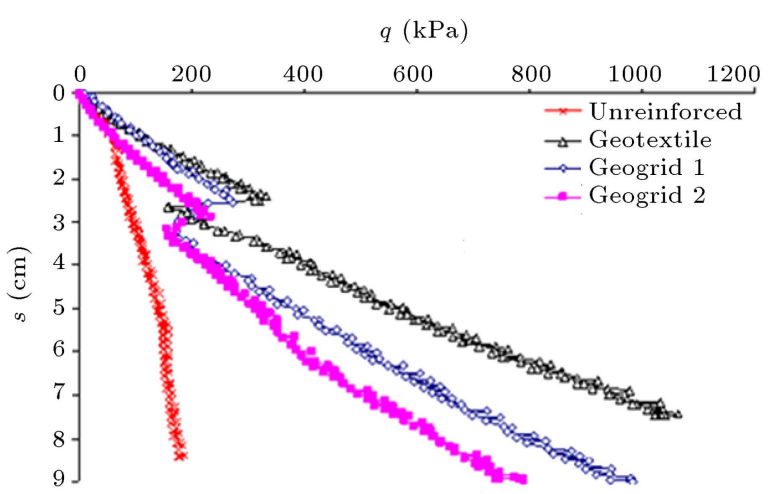

Figure 7. Load-settlement behavior of different reinforcement types for $u / B=0.75$.

values suddenly decrease at approximately $s / B=0.25$. As loading continues, the load values start to increase. However, the load-settlement curve for $u / B=0.75$ undergoes a sudden decrease. A possible reason for this behavior is that triangular failure surface is on the reinforcement line for greater first reinforcement depths such as $u / B=1$. Yet, this failure surface touches the reinforcement for lower first reinforcement depth values such as $u / B=0.75$. However, the depth is not enough to carry the load and the loading curve suddenly decreases. However, as is known, the sol used in the study is not dense. Therefore, when the model is loaded, the soil becomes denser and reinforcement starts to behave more effectively, thereby increasing the loading values for load-settlement curve. Based on Figure 7, the curve line of load-settlement for geotextile reinforcement is steeper. Table 1 shows that geotextile and Geogrid 2 have more or less similar tensile strength values, and their tensile strengths are greater than Geogrid 1. However, the geotextile has greater bearing ratios than the other reinforcements for $u / B=0.75$, as can be seen in Figure 7 . The interpretation of this behavior is that the soil-reinforcement contact surface is much larger for the geotextile. Since the soil is a medium sand, the interlocking of the geogrid cannot be sufficient enough for coarser particles. For different $u / B$ ratios, the number of reinforcement layers affects the bearing ratio values (Figure 8 ). Tests with geogrid reinforcements for small settlement ratios, such as $s / B=0.1$, had similar bearing ratios $(B R)$ at different first reinforcement depths. Geotextile and geogrid reinforcements show different maximum bearing ratios at different first reinforcement depths. When settlement ratios increase, maximum $B R$ values increase as the number of reinforcement layer increases. Settlement ratios become an important parameter on the bearing ratio values for multiple layered reinforcements and for the first reinforcement depth. For greater settlement ratios, this behavior changes. As settlement ratios increase, the differences between the bearing ratio values for each number of reinforcement layers decrease via different $u / B$ ratios. The bearing ratio and number of reinforcement layer relationship change for different $u / B$ values. The type of the load-settlement curve line begins to be linear.

The first reinforcement depth can affect the behavior of footings in the reinforced soil significantly. An optimum depth can lead to significant improvement through reinforcements. In the literature, generally, one type of reinforcement was used to investigate the effects of reinforcement [32]; however, in this study, the tests were conducted using different types of reinforcement materials. For geotextile, both geogrids behave perfectly when the first reinforcement layer is almost at $u=B / 2$, although, for the geotextile, the number of reinforcement layers, which changes the optimum depth value, is the same. Additionally, for the same number of reinforcement layers, the models reinforced with geogrids behave similarly when the first reinforcement layers change. However, the samples reinforced with the geotextile behave differently from other geogrids. Although the geotextile reinforcement has the maximum tensile strength (Table 1) as compared to 


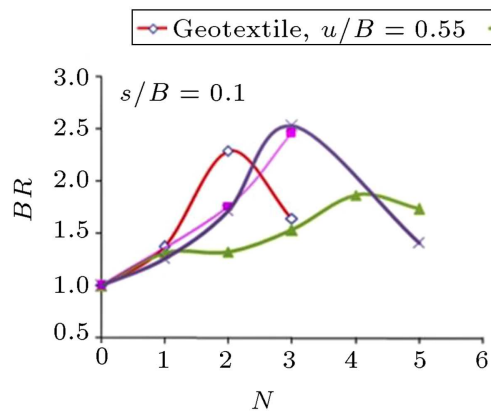

(a)

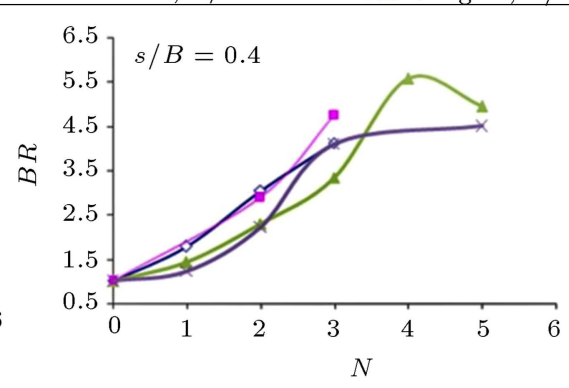

(b)

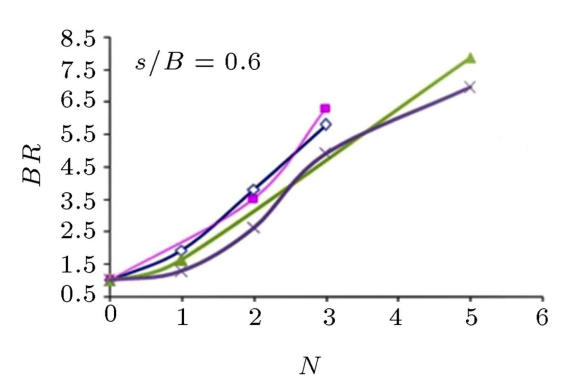

(c)

Figure 8. Bearing ratio-number of reinforcement layers behavior for different $u / B$ ratios: (a) $s / B=0.1$, (b) and $s / B=0.4$, and (c) $s / B=0.6$.

the geogrids, the latter have greater improvement effect than the geotextile with respect to bearing ratio values, because the aperture sizes of the geogrids improve the friction behavior between the soil and reinforcements. Additionally, the soil particles can fill the aperture gaps and, thus, behave more efficiently than the geotextile. However, for $u=0.75 B$, the model reinforced with geotextile has higher impact on the bearing ratios. Due to this different behavior, it can be assumed that the geotextile layer behaves as a rigid plate for greater depths and may restrain settlements. Furthermore, there can be no enough compaction effect by footing loading to fill the soil particles into apertures of the geogrids. For real projects, the geotextile can lead to higher improvement effect for higher first reinforcement depths such as $u=0.75 \mathrm{~B}$.

Another interesting point is that, generally, Geogrid 1 has greater improving effect than Geogrid 2, though Geogrid 1 has bigger tensile strength than Geogrid 2. However, Geogrid 1 has an aperture size twice smaller than Geogrid 2, meaning that when the soil particles can fill the smaller aperture sizes of the reinforcement, they can connect and increase the taking loads. Therefore, this behavior is important for real projects, because the behavior can be changed by different projects. To illustrate, a building or road projects with different reinforcements are required due to the particle size of subbase materials. Therefore, the particles and aperture sizes of the geogrids or scale effects of the materials used in the field should be considered.

Based on the test results, small and bigger settlement ratios are important for improvement effects of different reinforcement types. If the small settlement rates are important for a project, material types of the reinforcements are of greater importance than bigger settlement ratios might be, because the settlement rate increases different types of reinforcements, such as geotextile, and geogrids start to behave similarly.

The current study implies that, after footing settles to about $s=2.5 \mathrm{~B}$, all models show a sudden decrease in loading. However, after the settlement ratio reaches almost $s=3 B$, the geotextile and both geogrid reinforced models can be loaded, and the models have various linear load-settlement behaviors. Therefore, it may be assumed in this study that, prior to the real project, e.g., before constructing a building or road, a preloading department can be prepared. After loading of the reinforced field and the desired uniform settlements, the intended structure can be constructed on the preloaded reinforced field. Thus, more bearing ratios can be obtained.

\subsection{Finite-element model results}

To compare the results of the laboratory tests and understand the behaviour of the models, finite-element analyses were conducted. In order to verify the finite-element model, firstly, the unreinforced case was modelled. The load-settlement curves are observed in Figure 9. It can be seen that, for small settlement values, the curves behave almost similarly (until about $s / B=0,15)$. That is to say, the measured values from the experimental test and the load-settlement curves obtained from FEM agree well [10]. However, for bigger loading values, the differences start to be seen for two different methods. Thus, it can be estimated that, for medium dense conditions, small settlement ratios can be simulated by FEM easily; however, after the settlements and larger loadings, the soil starts to compact by footing loading, and upper soil parts become denser; then, its mechanical behaviour changes. In this respect, this case is one of the limitations of

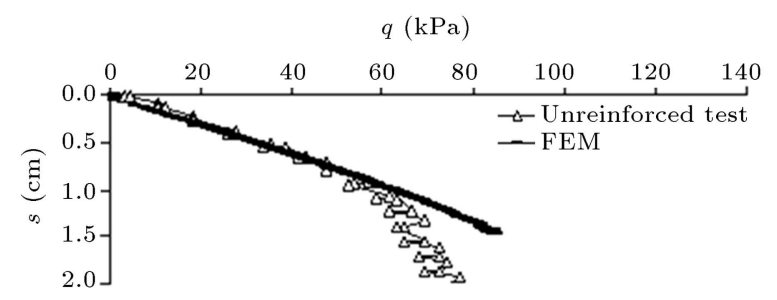

Figure 9. Comparison of FEM analysis and test measurements for unreinforced sand soil. 


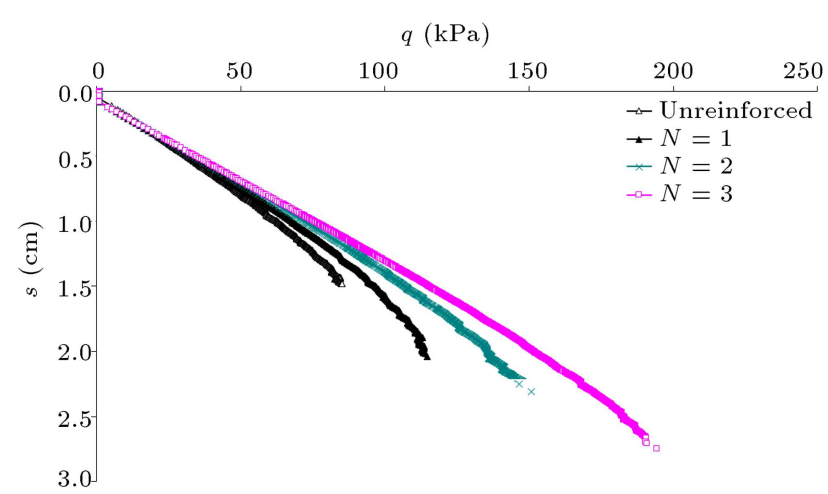

Figure 10. Behaviors of different reinforcement layers for finite-element models.

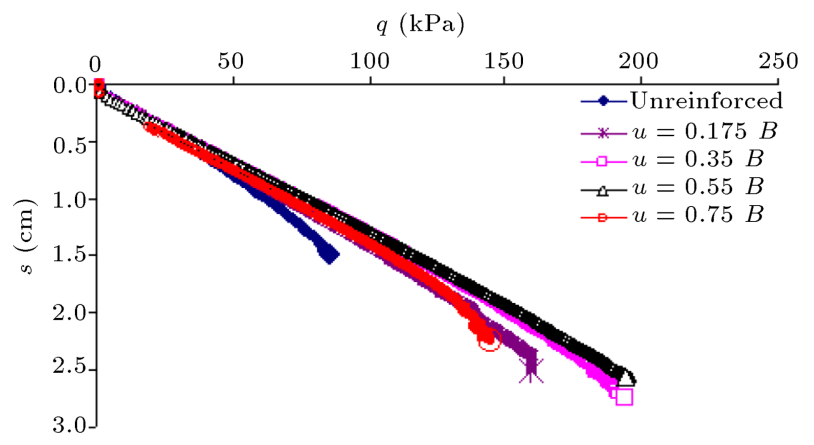

Figure 11. Load-settlement behavior of different reinforcement layers for FEM.

the FEM analysis; thus, it can be modelled for small settlements in this study.

Another limitation of the 2D modelling is the inability of determining the reinforcement materials, which have different aperture size properties. For 2D analysis, the aperture properties and their interaction between the soil particles cannot be modelled in the same conditions with the real laboratory tests. Therefore, in this study, only geotextile reinforced model was analyzed.

For reinforced models, different reinforcement layers were analyzed and their effects on the improvement of mechanical behavior were studied. Figure 10 illustrates the load-settlement curves for different reinforcement layers found by finite-element models. The number of reinforcement layers increases the loading, and bearing capacity of the model increases, too. The maximum improvement influence can be obtained by multi-layered reinforcement conditions for the threereinforcement-layered model $(N=3)$. Therefore, the number of reinforcements was $N=3$, and analyses were conducted until a settlement ratio reached $s / B=0.1$, because small settlement conditions can be compared with test results. The load settlement curves were analyzed for different first reinforcement depths, as can be observed in Figure 11, and the bearing ratio values $(B R)$ were compared, as shown in Figure 12. As the loading and settlement increase, the behaviors

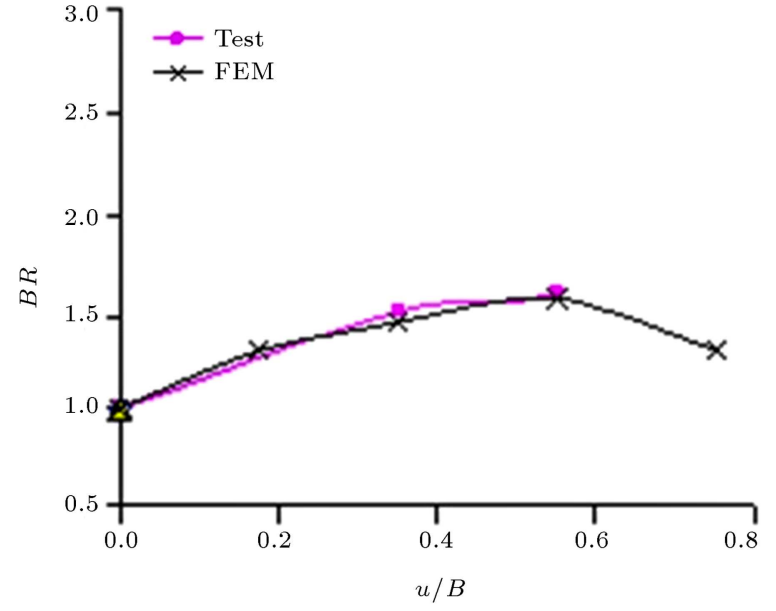

Figure 12. Comparison between FEM and test results for bearing ratio-first reinforcement depth.

of different models start to change by the effect of the first reinforcement depth. Thus, good agreement between FEM and geotextile reinforced results was also obtained for small settlement ratio values.

In order to understand the reason for the sudden decrease in $u=0.75 \mathrm{~B}$ in the laboratory tests, FEM analyses were conducted. In these analyses, since the small loading and settlement ratios can be compared with the test results, the loading value of $q=50 \mathrm{kPa}$ was used. The results of unreinforced and reinforced models were compared in terms of the failure wedge for stress behaviors in the reinforced models (Figure 13). The real figures were cut and zoomed to show the difference of the stress distributions under the footing. The scale is the same, but only changes in the stress are shown. The dark color parts show that there is no settlement and stress distribution. In this way, it can be also said that the boundary conditions are suitable. The stresses occur under the footing plate. The fluctuation in the reinforcements can determine the loads of each reinforcement and divide them into parts. In unreinforced soil, the triangular failure wedge can be seen in Figure 13(a) and can be observed as a normal behavior. However, for $u / B=0.175$ or the first smaller reinforcement depth, the failure plane has to pass through many reinforcement layers; however, for $u / B=0.75$ or the first greater reinforcement depth, the failure plane's less reinforcement layers intersect with each other (Figure 13(b) and (c)). Therefore, it can be interpreted that the reason for the sudden change observed in the load-settlement curve for $u / B=0.75$ is that when the reinforcement lies below this depth, the failure wedge of the soil does not intersect with many reinforcement layers; therefore, the contribution of the reinforcement is diminished. After some further settlement, the stresses are transferred to lower layers and, therefore, the reinforcements start to function again. Consequently, suddenly, the inclination 


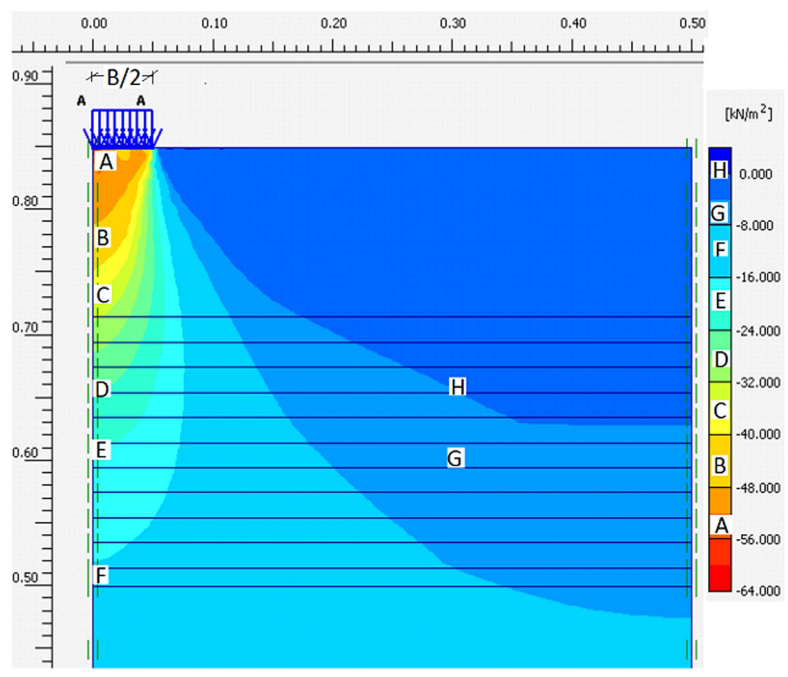

(a)

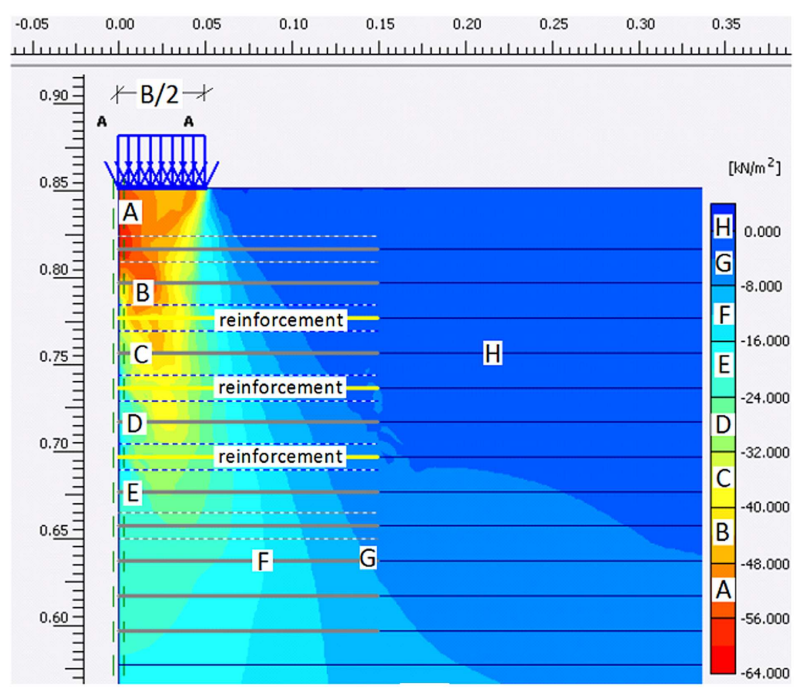

(b)

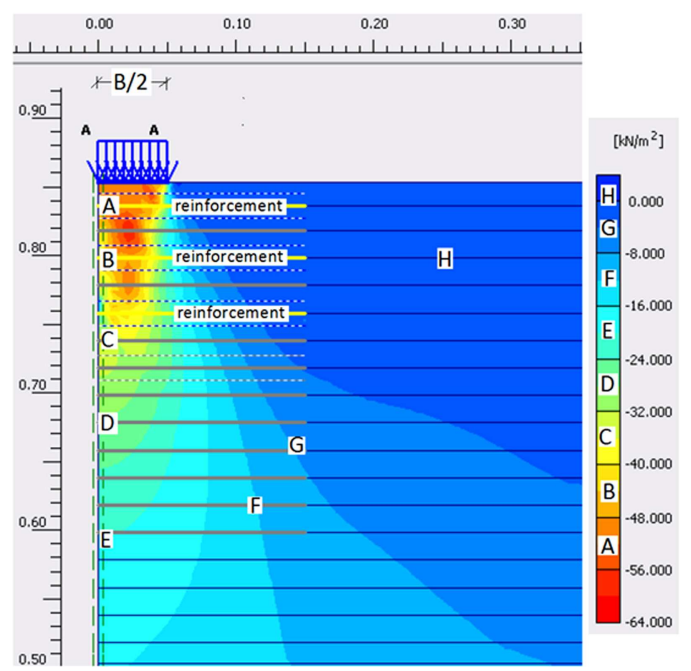

(c)

Figure 13. Behaviors of models for different first reinforcement ratios: (a) Unreinforced model, (b) $u / B=0.75$, and (c) $u / B=0.175$. of the load-settlement curve changes. Thus, the figure can explain the reason for the sudden decrease.

\section{Conclusion}

The laboratory model experiments and finite-element model analyses were carried out to determine the effect of the first reinforcement depth. The reinforcements included a geotextile and two different geogrids. The results indicated that the behavior changes depended on whether the settlement ratio is small or large. Laboratory test results of geotextile reinforced models were also compared to the results of finite-element model analysis. Based on the results obtained from the present study, many new and useful conclusions were drawn. These conclusions on the effects of the first reinforcement depth for reinforced medium dense sand are summarized below:

- This study shows that the first reinforcement depth has an important role in bearing ratio of footings on reinforced soils. It can be said that effective depth of the first reinforcement layer is approximately half of the footing width $(u=B / 2)$;

- As is expected, the geotextile reinforced system behaves differently from the geogrids;

- Different settlement ratios have important roles in reinforced soil performances;

- As settlement ratios increase, the bearing ratio values increase; however, the difference for all combinations of $u / B$ ratios and number of reinforcement layer decreases;

- For larger settlement ratios, the bearing rationumber of reinforcement layer relation changes for different $u / B$ values;

- Aperture size of reinforcement materials is important for interlocking effect to improve the bearing ratio values, although the reinforcement with smaller tensile strength can increase the mechanical behavior by interlocking effect. Hence, the tensile strength cannot be the only determinant to determine the efficient reinforcement;

- The settlement rate increases different types of reinforcements, such as geotextile, and geogrids start to behave similarly. This behavior can be significant for choosing the economical reinforcement type;

- The simple finite-element model used in this study showed that it could be used to model unreinforced and reinforced soil models very well at small settlement ratios. Since the settlements have to be limited in a civil engineering structure design, it can be stated that the finite-element model may be used successfully to model the reinforced subbase; 
- As the settlement ratio of the footing increases, different reinforcement types can achieve almost similar bearing ratios. Therefore, it may be considered that if a medium dense sand foundation soil is reinforced with a geosynthetic reinforcement and if the structure is placed upon it, the medium dense soil compacts under the load and the reinforcement will function even better. In other words, by preparing the preloading effect before the construction of the structure, more loading values can be obtained.

\section{Acknowledgement}

The authors thank the Scientific and Technical Research Council of Turkey (TUBITAK) and the Bogazici University Research fund.

\section{References}

1. Al-Qadi, I.L., Brandon, T.L., and Bhutta, S.A. "Geosynthetic stabilized flexible pavements", Proceedings of the Conference Geosynthetics, Long Beach, CA, USA, pp. 647-661 (1997).

2. Cancelli, A. and Montanelli, F. "In-ground test for geosynthetic reinforced flexible paved sand soils", Proceedings of the Conference Geosynthetics, Boston, MA, USA, pp. 863-878 (1999).

3. Perkins, S.W. "Evaluation of geosynthetic reinforced flexible pavement systems using two pavements test facilities", Federal Highway Administration Report FHWA/MT-02-008/20040, Montana Department of Transportation, Helena, MT, USA, p. 120 (2002).

4. Hufenus, R., Rueegger, R., Banjac, R., Mayor, O., Springman, S.M., and Bronnimann, R. "Full-scale field tests on geosynthetic reinforced sand soils on soft subgrade", Geotextiles and Geomembranes, 24(1), pp. 21-37 (2006).

5. Abu-Farsakh, M.Y. and Chen, Q. "Evaluation of geogrid base reinforcement in flexible pavement using cyclic plate load testing", International Journal of Pavement Engineering, 12(3), pp. 275-288 (2011).

6. Abu-Farsakh, M.Y., Akond, I., and Chen, Q. "Evaluating the performance of geosynthetic-reinforced sand soils using plate load tests", International Journal of Pavement Engineering, 17(10), pp. 901-912 (2016).

7. Badakhshan, E. and Noorzad, A. "Effect of footing shape and load eccentricity on behavior of geosynthetic reinforced sand bed", Geotextiles and Geomembranes, 4(5), pp. 58-67 (2017).

8. Moffat, R. Jadue, C., Beltran, J.F., and Herrera, R. "Experimental evaluation of geosynthetics as reinforcement for shotcrete", Geotextiles and Geomembranes, pp. 1-8 (2017).

9. Kargar, M., Mir Mohammad Hosseini, S.M. "Effect of reinforcement geometry on the performance of a reduced-scale strip footing model supported on geocellreinforced sand", Scientia Iranica A, 24(1), pp. 96-109 (2017).
10. Cicek, E. "Analysis of strip plates on geosynthetic reinforced sand", Ph.D. Dissertation, Institute of Science in Ataturk University, Erzurum, Turkey (2011).

11. Pasquini, E., Bocci, M., Ferrotti, G., and Canestrari, F. "Laboratory characterization and field validation of geogrid-reinforced asphalt pavements", Road Materials and Pavement Design, 14(1), pp. 17-35 (2013).

12. Nair, A.M. and Latha, G.M. "Repeated load tests on geosynthetic reinforced sand soil sections", Geomechanics and Geoengineering: An International Journal, 11(2), pp. 95-103 (2016).

13. Tang, X., Palomino, A.M., and Stoffels, S.M. "Permanent deformation behavior of reinforced flexible pavements built on soft soil subgrade", Road Materials and Pavement Design, 17(2), pp. 311-327 (2016).

14. Tuna, S.C. and Altun, S. "Mechanical behaviour of sand-geotextile interface", Scientia Iranica A., 19(4), pp. 1044-1051 (2012).

15. Sobhan, K. and Tandon, V. "Mitigating reflection cracking in asphalt overlay using geosynthetic reinforcements", Road Materials and Pavement Design, 9(3), pp. 367-387 (2008).

16. Fallah, S. and Khodaii, A. "Developing a fatigue fracture model for asphalt overlay reinforced with geogrid", Materials and Structures, 49, pp. 1705-1720 (2016).

17. Canestrari, F., Grilli, A., Santagata, F.A., and Virgili, A. "Interlayer shear effect of geosynthetic reinforcements", Proceedings of the 10th International Conference on Asphalt Pavements, Quebec City, pp. 811-820 (2006).

18. Komatsu, T., Kikuta, H., Tuji, Y., and Muramatsu, E. "Durability assessment of geogrid-reinforced asphalt concrete", Geotextiles and Geomembranes, 16(5), pp. 257-271 (1998).

19. Webster, S.L. "Geogrid reinforced base courses for flexible pavements for light aircraft: test section construction, behavior under traffic, laboratory tests, and design criteria", Technical Report GL-93-6, USAE Waterways Experiment Station, Vicksburg, MS, USA, p. 86 (1993).

20. Al-Qadi, I.L., Dessouky, S., Kwon, J., and Tutumluer, E. "Geogrid in flexible pavements: validated mechanism", Transportation Research Record: Journal of the Transportation Research Board, 2045, National Research Council, pp. 102-109 (2008).

21. Hass, R., Walls, J., and Carroll, R.G. "Geogrid reinforcement of granular bases in flexible pavements", Transportation Research Record: Journal of the Transportation Research Board, 1188, pp. 19-27 (1988).

22. Guido, V.A., Chang D.K., and Sweeney M.A. "Comparison of geogrid and geotextile reinforced earth slabs", Canadian Geotechnical Journal, 23(4), pp. 440435 (1986).

23. Chen, Q. "An experimental study on characteristics and behavior of reinforced soil foundation", $\mathrm{PhD}$ Dissertation, Louisiana State University, US (2007). 
24. Mandal, J.N. and Manjunath, V.R. "Bearing capacity of strip plate resting on reinforced sand subgrades", Construction and Building Materials, 9(1), pp. 35-38 (1995).

25. Dash, S.K., Krishnaswamy, N.R., and Rajagopal, K. "Bearing capacity of strip plates supported on geocellreinforced sand", Geotextiles and Geomembranes, 19, pp. 235-256 (2001).

26. Alamshahi, S. and Hataf, N. "Bearing capacity of strip plates on sand slopes reinforced with geogrid and gridanchor", Geotextiles and Geomembranes, 27, pp. 217226 (2009).

27. Chakraborty, M. and Kumar, J. "Bearing capacity of circular foundations reinforced with geogrid sheets", Soils and Foundations, 54(4), pp. 820-832 (2016).

28. Dawson, A.R. and Moghaddas Tafreshi, S.N. "Comparison of bearing capacity of a strip plate on sand with geocell and with planar forms of geotextile reinforcement", Geotextiles and Geomembranes, 28, pp. 72-84 (2010).

29. El Sawwaf, M. and Nazir, A.K. "Behavior of repeatedly loaded rectangular plates resting on reinforced sand", Alexandria Engineering Journal, 49(4), pp. 349-356 (2010).

30. Shin, E.C., Das, B.M., Lee, E.S., and Atalar, C. "Bearing capacity of strip foundation on geogrid-reinforced sand", Geotechnical and Geological Engineering, 20(2), pp. 169-180 (2002).

31. Cicek, E., Guler, E., and Yetimoglu, T. "Comparison of measured and theoretical pressure distribution below strip footings on sand soil", International Journal of Geomechanics, 14(5), p. 4 (2014).

32. Harikumar, M., Sankar, N., and Chandrakaran, S. "Behaviour of model footing resting on sand bed reinforced with multidirectional reinforcing elements", Geotextiles and Geomembranes, 44, pp. 568-578 (2016).

\section{Biographies}

Elif Cicek received her $\mathrm{PhD}$ degree in Civil Engineering at Ataturk University, Turkey in 2011. She took many courses in Istanbul Technical University and Marmara University, Turkey. Her thesis' numerical and experimental studies were conducted at Bogazici University, Turkey and was supported with doctorate scholarship by Scientific and Technical Research Council of Turkey (TUBITAK). She is working as Asst. Prof. Dr. in Hacettepe University since 2015. Her research interests include effects of geosynthetic reinforcements, pressure distribution of soils, slope stability, earthquake, limit equilibrium approaches, numerical methods, transportation geotechnic, traffic engineering, highway design, material properties, concrete roads, numerical calculations, limit equilibrium methods, etc.

Erol Guler is a Professor of Civil Engineering at Bogazici University, Istanbul, Turkey. He is an IGS Council member. He founded the IGS Turkish Chapter in 2001 and served as its president until 2005 and was re-elected as President again in 2011. He represents Turkish Standards Institute (TSE) at the International Standards Organization (ISO) and the European Committee for Standardisation (CEN). He is the Convener of the WG2 of the ISO TC221 (Technical Committee on geosynthetics) and is also the Convener of the WG2 of the CEN TC189 (Technical Committee on geosynthetics). He is currently an international member of the USA TRB Committee on geosynthetics. His research subjects include mainly geosynthetics and specifically geosynthetic reinforcement and liner systems. He enjoys vast experience in design of foundations, deep excavations, landslide mitigation and site investigations.

Temel Yetimoglu is an Emeritus Professor of Civil Engineering in Ataturk University, Erzurum, Turkey. He obtained his $\mathrm{PhD}$ degree in Istanbul Technical University. He has many academic studies in the geotechnical engineering field. Some of his research areas are reinforced soils, piles, numerical analyses, plate load tests, geosynthetics, laboratory experiments, and environmental geotechnic. 